\title{
A pure tone audiogram for students
}

Keywords: audiogram, hearing impairment, ear trauma, sensorineural hearing loss, mastoid bone, rinne test, chronic otitis media, auditory meatus

Abbrevations: SNHL, sensorineural hearing loss; CHL, conductive hearing loss; $\mathrm{AC}$, air conduction; $\mathrm{BC}$, bone conduction; $\mathrm{MB}$, mastoid bone; AC: auditory meatus

\section{Case summary}

A 78year old Caucasian female attends an otology clinic with a 3-week history of right ear discharge associated with minimal discomfort. She also reports worsening of her known hearing impairment. She uses a hearing aid in the right ear. She has a long standing significant hearing loss affecting the left ear. She denies any history of vertigo, tinnitus, ear trauma or previous ear surgery.

On examination there is ongoing right sided acute otitis externa complicated by a wet perforated tympanic membrane. The left ear is essentially normal. After tuning fork tests, the patient undergoes pure tone audiogram which can be seen in Figure 1.

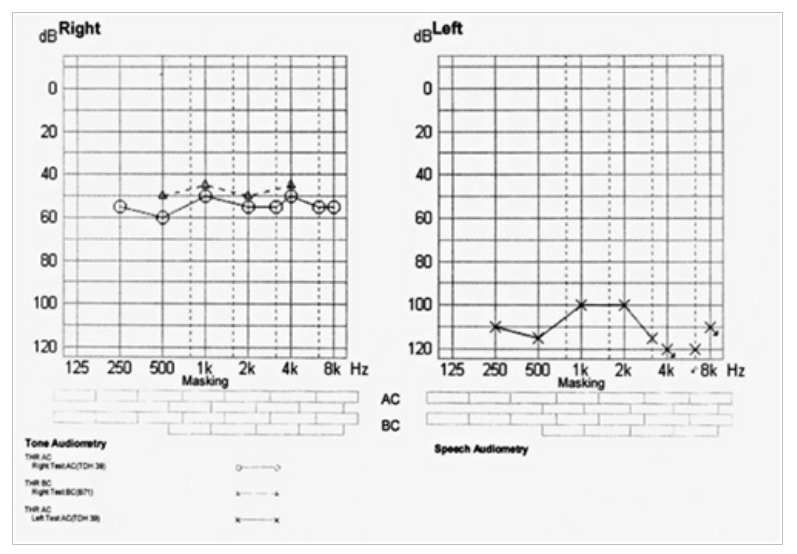

Figure I Pure tone audiogram.

\section{Questions}

a. What is a pure tone audiogram?

b. How is an audiogram interpreted?

c. What does the audiogram in Figure 1 show?

d. What additional tests can aid in diagnosing hearing loss?

\section{Answers}

a. A pure tone audiogram is a graphic representation of air and bone conduction of sound. Audiograms can be very useful to the clinician in order to assess a patient's hearing. There are three main types of deafness: sensorineural, conductive and mixed. Sensorineural hearing loss (SNHL) results secondary
Volume 5 Issue I - 2016

\author{
Paul Francis McLean, Muhammad Shakeel \\ Ninewells hospital University of Dundee, UK
}

Correspondence: Paul Francis McLean Final year medical student University of Dundee Dundee DDI 4HN, UK, Email p.f.mclean@dundee.ac.uk

Received: September 04, 2016 | Published: December 07, 2016

to a defect within the inner ear, notably the cochlea, cochlear nerve or-infrequently-central pathways. ${ }^{1}$ Conductive hearing loss (CHL) involves a defect in the outer or middle ear. Mixed hearing loss is a combination of these pathologies. A pure tone audiogram, such as that seen in Figure 1, can identify which type of deafness a patient has. This is a subjective test that relies on the patient's responses to stimuli. The audiogram examines air conduction (AC) and bone conduction (BC). To measure AC a series of sounds, which are preset at particular frequencies, are played via headphones or insert phones to the patient, who is advised to inform the examiner whether or not they can hear these sounds. Similarly, BC is measured by placing a bone vibrator on the mastoid process whilst masking the ear which is not being examined with a continuous pure tone in order to avoid crosstransmission ${ }^{2}$. The results are plotted in a graph and are then interpreted by the examiner.

b. Plotted on the x-axis is frequency of sound, from low to high. The $y$-axis represents the level of sound, measured in decibels (dB), with the level decreasing as the axis ascends. The $\mathrm{dB}$ scale is a logarithmic one, which means that a step up of $10 \mathrm{~dB}$ equates to 10 -fold increase of the actual sound pressure used. Remember that the decibel recording is the first volume that the patient can hear, therefore the higher the decibel recorded the worse the hearing loss is. The following keys explain the notation of an audiogram. o-Air conduction (right ear); x-Air conduction (left ear); $\Delta$-Unmasked bone conduction; -Masked bone conduction (right ear); -Masked bone conduction (left ear).

"Normal hearing" is defined as having both air and bone conduction readings of 20 decibels or better (i.e. lower than 20). Beyond this, hearing loss is categorised into mild (20-40), moderate (40-70), severe (70-95) and profound (95 and above). ${ }^{3}$ 
With a conductive hearing loss, there is normal masked bone conduction but a decrease in air conduction. The difference between the air and masked bone conductions (air-bone gap) should be at least 10 decibels for a diagnosis of conductive hearing loss to be made. This is secondary to a problem in the transmission of the sound from the outer or/and middle ear to the cochlea. Some of the causes include wax impaction, tympanic membrane perforation, glue ear, and otosclerosis. An example of sensorineural hearing loss would be Presbycusis which is the result of age related loss of hair cells. Both air and masked bone conduction are worse than 20 decibels in a sensorineural hearing loss, but there is no considerable air-bone gap present. With a mixed hearing loss, masked bone-conduction is worse than 20 decibels, and there is also an air-bone gap of greater than 10 decibels. $^{3}$

c. The audiogram reveals a profound sensorineural hearing loss (SNHL) in the left ear and a moderate, mainly sensorineural loss on the right side. The clinical picture is complicated by two concomitant pathologies: right ear infection, making it difficult for the patient to wear the hearing aid; and a profound SNHL in left ear, unsuitable for a hearing aid.

d. In addition to the audiogram, the clinician can carry out some simple examinations in order to help diagnose and classify hearing loss. Two routine tests performed are Rinne and Weber tests. It is important that medical students and junior doctors are competent in carrying out these examinations. The Weber test is useful for a conductive as well as a sensorineural hearing loss. If the patient lateralizes the sound to his better hearing ear, it is a SNHL, if he hears it in his worse ear it is a CHL. Experienced clinicians would usually do the Weber test first as it is the quickest way to distinguish between a unilateral CHL and SNHL. The Rinne test is valuable as a confirmation and also in the special situation of a bilateral CHL with a central Weber test. The Rinne test is used to test for conductive hearing loss and involves placing a high frequency tuning fork $(512 \mathrm{~Hz})$ against the mastoid bone $(\mathrm{BC})$ and asking the patient to say when he/she stops hearing it-when the patient answers, the tuning fork should be moved quickly to just outside the auditory meatus (AC), and the examiner should ask if the sound reappears. If the sound reappears, and $\mathrm{AC}>\mathrm{BC}$, this can be thought of as a normal response to the Rinne test (Rinne positive). Conversely, if $\mathrm{BC}>\mathrm{AC}$ is abnormal, this is indicative of a conductive deficit (Rinne negative). The Weber test involves placing a $512 \mathrm{~Hz}$ tuning fork in the middle of the forehead of a patient and asking the patient to indicate to which ear the sound is heard louder. Ideally the noise should be heard in the middle of the head; or in other words, if both ears hear the tuning fork vibrations equally well. However, if Weber lateralizes to the right ear then there are two interpretations: the right ear has a conductive hearing loss, or the left ear has a SNHL hearing loss. The Rinne test helps clarify the situation; as the $\mathrm{BC}$ will be better than the $\mathrm{AC}$ in conductive loss, while the $\mathrm{AC}$ will be better than the $\mathrm{BC}$ in SNHL.

\section{Patient's outcome}

This patient presented with otitis externa and ear discharge secondary to chronic otitis media, thus making it difficult for her to wear a hearing aid. The priority here is to treat the right ear infection with microsuction clearance followed by topical antibiotic ear drops containing a steroid. The left ear problem is long standing and no active intervention is required at present.

\section{Acknowledgments}

None.

\section{Conflicts of interest}

Author declares there are no conflicts of interest.

\section{Funding}

None.

\section{References}

1. Collier J, Longmore M, Amarakone. Oxford Handbook of Clinical Specialties. (9th edn), Oxford, UK. 2013.

2. Bull P, Clarke R.Lecture Notes: Diseases of the Ear, Nose and Throat. (10th edn), Oxford. Blackwell, UK. 2007.

3. Tysome JR, Kanegaonkar R. ENT: An Introduction and Practical Guide. (1st edn), Ch10. Taylor \& Francis Ltd, UK. 2011. p. 1-176. 